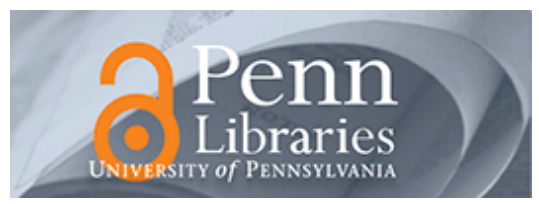

University of Pennsylvania ScholarlyCommons

October 2006

\title{
Regions that influence acoustic propagation in the sea at moderate frequencies, and the consequent departures from the ray-acoustic description
}

John L. Spiesberger

University of Pennsylvania, johnsr@sas.upenn.edu

Follow this and additional works at: https://repository.upenn.edu/ees_papers

\section{Recommended Citation}

Spiesberger, J. L. (2006). Regions that influence acoustic propagation in the sea at moderate frequencies, and the consequent departures from the ray-acoustic description. Retrieved from

https://repository.upenn.edu/ees_papers/45

Copyright ASA. Reprinted from Journal of the Acoustical Society of America, Volume 120, Issue 4, October 2006, pages 1842-1850.

Publisher URL: http://dx.doi.org/10.1121/1.2336991

This paper is posted at ScholarlyCommons. https://repository.upenn.edu/ees_papers/45

For more information, please contact repository@pobox.upenn.edu. 


\title{
Regions that influence acoustic propagation in the sea at moderate frequencies, and the consequent departures from the ray-acoustic description
}

\author{
Abstract \\ In the limit where a transient signal is comprised of very large frequencies, spatial regions within an \\ inhomogeneous medium that influence the propagation from a source to a receiver lie along one or more \\ ray paths. At lower frequencies for which the geometrical acoustic approximation is of borderline \\ applicability, the regions that influence such transient signals are extended because of diffraction. \\ Previous research has addressed the numerical determination of those spatial regions that influence \\ propagation at low frequency. The present paper addresses the question of how high the center \\ frequency need be so that the regions of influence are nearly described as ray paths for a model ocean in \\ which the speed of sound increases nearly linearly with depth from a perfectly reflecting surface. \\ Computations indicate that near $2500 \mathrm{~Hz}$ and at a range of $50 \mathrm{~km}$, the region of influence resembles a ray. \\ Noticeable departures from the ray picture are found at a range of $500 \mathrm{~km}$. Various physical and \\ mathematical causes for the departures from the ray propagation model for lower frequencies and for \\ greater ranges are identified and discussed.

\section{Comments} \\ Copyright ASA. Reprinted from Journal of the Acoustical Society of America, Volume 120, Issue 4, \\ October 2006, pages 1842-1850. \\ Publisher URL: http://dx.doi.org/10.1121/1.2336991
}




\title{
Regions that influence acoustic propagation in the sea at moderate frequencies, and the consequent departures from the ray-acoustic description
}

\author{
John L. Spiesberger \\ Department of Earth and Environmental Science, 240 S. 33rd Street, University of Pennsylvania, \\ Philadelphia, Pennsylvania 19104-6316
}

(Received 4 April 2004; revised 23 July 2006; accepted 24 July 2006)

\begin{abstract}
In the limit where a transient signal is comprised of very large frequencies, spatial regions within an inhomogeneous medium that influence the propagation from a source to a receiver lie along one or more ray paths. At lower frequencies for which the geometrical acoustic approximation is of borderline applicability, the regions that influence such transient signals are extended because of diffraction. Previous research has addressed the numerical determination of those spatial regions that influence propagation at low frequency. The present paper addresses the question of how high the center frequency need be so that the regions of influence are nearly described as ray paths for a model ocean in which the speed of sound increases nearly linearly with depth from a perfectly reflecting surface. Computations indicate that near $2500 \mathrm{~Hz}$ and at a range of $50 \mathrm{~km}$, the region of influence resembles a ray. Noticeable departures from the ray picture are found at a range of $500 \mathrm{~km}$. Various physical and mathematical causes for the departures from the ray propagation model for lower frequencies and for greater ranges are identified and discussed. (C) 2006 Acoustical Society of America. [DOI: 10.1121/1.2336991]
\end{abstract}

PACS number(s): 43.20.El [ADP]

Pages: $1842-1850$

\section{INTRODUCTION}

Quantifying where a wavelike signal is influenced between a source and receiver by a medium and its fluctuations is useful in acoustics, communication, scattering theory, optics, and fluid and cosmic gravity waves problems. At infinite frequency, the received signal is influenced only on one or more infinitesimally thin rays. At finite frequencies, the notion of influence is often quantified by considering experiments in which a screen is placed between a source and receiver. For homogeneous media, one imagines increasing the radius of a circular opening in the screen until the received signal is similar to that obtained without a screen. For transmission at a single frequency, the first Fresnel zone provides a radius that approximately admits a field similar to that found without a screen. ${ }^{1}$ There remains a bright spot at the center that reduces to that found without a screen when the radius is much larger. ${ }^{1}$ When a signal has a nonzero bandwidth, the free-field solution is obtained exactly, within a pulse resolution of the time of the direct arrival, when the radius is given by the zone of influence, ${ }^{2-6} R_{I}(x) \cong(2 c T x(d$ $-x) / d)^{1 / 2}$. Here, the speed of the wave is $c$, its temporal resolution is $T$, the distance between source and receiver is $d$, and $x$ measures distance from the source to receiver. Circular or otherwise shaped openings interfere with the transmitted signal, and in particular their edges lead to edge-diffracted rays. ${ }^{1,7}$ Such rays are entirely caused by diffraction and appear to exist for all edges even when the screen is perfectly absorbing. ${ }^{1,7}$ When the transmission consists of a single frequency, edge-diffracted rays cause the anomalously bright spot mentioned above. For transmissions with nonzero bandwidth, the edge-diffracted rays arrive exactly one pulse reso- lution later than the signal traveling directly between the source and receiver when the circular opening has radius given by the zone of influence.

For any transient signal at finite frequencies, an exact method has been developed to compute the region in any medium that significantly influences the received signal for any specified window of signal travel time. ${ }^{8}$ This window is sometimes chosen to surround a peak. Results at finite frequencies differ from those at infinite frequency because of diffraction. The purpose of this paper is to investigate how high the center frequency of a signal need be in an ideal oceanic acoustic waveguide to yield a region of influence that resembles one or more ray paths. For homogeneous media, the exact method is different than the idea of a zone of influence. Perhaps the most interesting difference is that the method allows one to look within the zone of influence to see at high spatial resolution how each region of space influences the signal. This high-resolution picture is possible to compute for inhomogeneous media as well. ${ }^{8}$

Solutions are given for 50- and 500-km ranges of propagation. A waveguide speed is chosen to increase nearly linearly with depth such that an analytical solution to the wave equation is available. Results based on the analytical solution are compared with those derived from the sound-speed insensitive parabolic approximation. ${ }^{9}$ If these results are similar, then results previously given for this approximation are probably accurate. $^{8}$

James Bowlin pioneered the use of the Huygens-Fresnel principle (pp. 370-375 in Ref. 1) to estimate paths of transient signals between a source and a receiver in the ocean. ${ }^{10}$ The results in Ref. 8 expand on his ideas in three ways. They are the following: (1) The integral theorem of Helmholtz and 
Kirchhoff is used to calculate effects of diffraction. This theory is more accurate than the Huygens-Fresnel principle. ${ }^{1}$ (2) A method is identified for obtaining quantitative values of medium influence on the received signal at high spatial resolution within the domain. (3) An interference filter is found useful for visualizing regions of space that significantly influence the received signal. The calculations in this paper use these three methods. We also compare results from the Huygens-Fresnel principle with those obtained from the integral theorem of Helmholtz and Kirchhoff.

\section{SUMMARY OF METHOD FOR ESTIMATING REGION OF INFLUENCE}

We summarize the methods ${ }^{8}$ used to compute a region of influence for a transient signal in any medium. Suppose an infinitely large opaque screen is perpendicular to the $x$ axis at $x_{s c}$. The screen is in-between a source and receiver. The $z$ axis is parallel to the screen. The integral theorem of Helmholtz and Kirchhoff ${ }^{1}$ yields the contribution to the time series at time $t$ at the receiver from the wave field passing through a transparent opening of the screen between coordinates $z_{r}$ and $z_{s}$

$$
e\left(t, x_{s c}, z_{r}, z_{s}\right)=B \int_{z_{r}}^{z_{s}} \int_{-\infty}^{\infty} H_{1}\left(x_{s c}, z, \omega\right) \exp (-i \omega t) d \omega d z,
$$

where $B$ is a normalization constant. The radian frequency is $\omega$ and

$$
\begin{aligned}
H_{1}\left(x_{s c}, z, \omega\right)= & W_{1}\left(x_{s c}, z, \omega\right) \frac{\partial W_{0}\left(x_{s c}, z, \omega\right)}{\partial x} \\
& -W_{0}\left(x_{s c}, z, \omega\right) \frac{\partial W_{1}\left(x_{s c}, z, \omega\right)}{\partial x} .
\end{aligned}
$$

The solutions of the Helmholtz equation on the screen due to emissions located at the source and receiver are $W_{0}\left(x_{s c}, z, \omega\right)$ and $W_{1}\left(x_{s c}, z, \omega\right)$ respectively. ${ }^{1}$ We will also work with

$$
G\left(t, x_{s c}, z_{r}, z_{s}\right)=\mathcal{F}\left[e\left(t, x_{s c}, z_{r}, z_{s}\right)\right],
$$

where $\mathcal{F}$ removes the carrier frequency via complex demodulation of analytic signals [Eq. (47) of Ref. 9]. Results using the Huygens-Fresnel principle ${ }^{1}$ with an inclination factor of unity can be obtained by substituting

$$
H_{2}\left(x_{s c}, z, \omega\right)=W_{0}\left(x_{s c}, z, \omega\right) W_{1}\left(x_{s c}, z, \omega\right),
$$

for $H_{1}\left(x_{s c}, z, \omega\right)$ in Eq. (1).

The "region of influence" denotes locations through which a transient signal travels that significantly affect the received signal within a specified window of travel time. This region is different than the concept for the zone of influence, which yields a time series identical to that found without a screen within the temporal resolution of the signal. For narrow-band signals in inhomogeneous media, it could be necessary to extend the region toward infinity to guarantee reception of an identical signal. Instead, the region of influence's boundaries demarcate locations of significant contribution.
A term is needed to specify what is being influenced in the received time series. We call it the "measure of influence." Two such measures are defined. The measure of influence of the first type is the largest peak in the time series obtained from apertures $z=0$ through $z=z_{j}$,

$$
M_{1}\left(t_{0}, x_{s c}, 0, z_{j}\right) \equiv \max _{t \in\left(t_{0} \pm \delta t / 2\right)}\left\{V\left(t, x_{s c}, 0, z_{j}\right)\right\} .
$$

where $V\left(t, x_{s c}, 0, z_{j}\right)$ is some function of the received time series within the window of travel time of duration $\delta t$. The measure of influence of the second type is the energy of the function of the time series in the window,

$$
M_{2}\left(t_{0}, x_{s c}, 0, z_{j}\right) \equiv \int_{t_{0}-\delta t / 2}^{t_{0}+\delta t / 2} V^{2}\left(t, x_{s c}, 0, z_{j}\right) d t .
$$

Examples of $V\left(t, x_{s c}, 0, z_{j}\right)$ are the time series with and without the carrier frequency [Eqs. (1) and (3)], and a time series that is adjusted for interference with a filter, $\mathcal{I}$,

$$
\int_{z_{r}}^{z_{s}} \int_{-\infty}^{\infty} \mathcal{I}\left\{H_{1}\left(x_{s c}, z, \omega\right)\right\} \exp (-i \omega t) d \omega d z,
$$

discussed later.

The "differential measure of influence" is obtained using a first difference of the measure of influence as

$$
\begin{aligned}
& \delta M_{k}\left(t_{0}, x_{s c}, z_{j}\right) \\
& \quad= \begin{cases}M_{k}\left(t_{0}, x_{s c}, 0, z_{1}\right) & \text { if } j=1, \\
M_{k}\left(t_{0}, x_{s c}, 0, z_{j}\right)-M_{k}\left(t_{0}, x_{s c}, 0, z_{j-1}\right) & \text { if } j>1,\end{cases}
\end{aligned}
$$

where $k=1,2$ denotes measures of the first and second types, respectively. The differential measure of influence contains the information needed to quantify the influence of the signal passing through any aperture of a screen on the received signal. However, when the received phase changes quickly from signals passing through nearby apertures, it can be difficult to visualize regions that yield a significant contribution. The interference filter is designed to make it easier to understand what is happening in this situation. The interference filter outputs only net positive contributions to the measure of influence. ${ }^{8}$ The region of influence so filtered is called the "net region of influence." ence filter is used here because it is more accurate than the unweighted filter. ${ }^{8}$

We choose boundaries for the region of influence by including significant contributions to the measure of influence. For any screen, the contributions from all apertures yield the final value for the measure of influence, $M_{i}\left(t_{0}, x_{s c}, 0, D\right)$, where the bottom of the screen is $z=D$. The absolute values of the differential measure of influence are sorted into decreasing order. The cumulative sum of the first $N$ sorted apertures is

$$
\mathcal{L}(N) \equiv \sum_{n=1}^{N} \frac{\left|\delta M_{k}\left(t_{0}, x_{s c}, z_{g(n)}\right)\right|}{\left|M_{k}\left(t_{0}, x_{s c}, 0, D\right)\right|}
$$

where $g(n)$ denotes sorted order. With the fractional amplitude method, we say that the measure of influence is reconstructed with a fidelity, $f$, by choosing the smallest value of 
$N=1,2,3, \ldots$ such that $\mathcal{L}(N)$ exceeds $f$ without any value $\mathcal{L}(N+p)$ being less than $f$ for integer $p$ greater than zero. For example, if we wish to reconstruct the measure of influence with a fidelity of $0.9, f$ is 0.9 . Perfect fidelity corresponds to $f=1$.

\section{SHORTER DISTANCE PROPAGATION}

A source and receiver are placed at a depth of $5 \mathrm{~m}$ and separated by $50 \mathrm{~km}$. The speed of sound is taken to increase with depth, $z$, almost linearly according to

$$
c(z)=c_{0} / \sqrt{1-2 a z}, \quad z \leqslant 1 /(2 a)
$$

because this yields an analytical solution ${ }^{11}$ to Helmholtz's equation in cylindrical coordinates,

$$
\begin{gathered}
\frac{\partial^{2} W(r, z, \omega)}{\partial r^{2}}+\frac{1}{r} \frac{\partial W(r, z, \omega)}{\partial r}+\frac{\partial^{2} W(r, z, \omega)}{\partial z^{2}} \\
+k^{2}(z) W(r, z, \omega)=-\frac{2}{r} \delta\left(z-z_{s}\right) \delta(r) .
\end{gathered}
$$

The depth of the source is $z_{s}$ and it is at a radius, $r$, of zero. The acoustic wave number is $k=\omega / c(z)$.

\section{A. Exact solution from normal modes}

Pressure perturbations vanish at the surface and at infinite $z$ so $W(r, 0, \omega)=0$ and $\lim _{z \rightarrow \infty} W(r, z, \omega)=0$. The solution ${ }^{11}$ to Helmholtz's equation is

$$
W(r, z, \omega)=\pi i \sum_{l=1}^{\infty} \frac{\operatorname{Ai}\left(z_{s} / H-y_{l}\right) \operatorname{Ai}\left(z / H-y_{l}\right) H_{0}^{(1)}\left(\xi_{l} r\right)}{\int_{0}^{1 / 2 a} \operatorname{Ai}^{2}\left(z / H-y_{l}\right) d z}
$$

where $i$ is $\sqrt{-1}, l$ is mode number, Ai is the Airy function given by Eq. (10.4.2) in Ref. 12, $-y_{l}$ is the $l$ th zero of the Airy function (all zeros are negative so $y_{l}$ is positive), $z_{s}$ is the depth of the acoustic source,

$$
\begin{aligned}
& H \equiv\left(2 a k_{0}^{2}\right)^{-1 / 3}, \\
& \xi_{l}^{2}=k_{0}^{2}-y_{l} / H,
\end{aligned}
$$

and $k_{0} \equiv \omega / c_{0}$. The time series at a receiver at cylindrical coordinate $\left(r_{s}, z_{r}\right)$ is obtained from the inverse Fourier transform of Eq. (12).

The group speed of mode $l$ is

$$
\begin{aligned}
c_{g}= & c_{0} \\
& \times\left(1-y_{l}\left(2 a c_{0}\right)^{2 / 3} \omega^{-2 / 3}\right)^{1 / 2}(1 \\
& \left.-2 y_{l}\left(2 a c_{0}\right)^{2 / 3} \omega^{-2 / 3} / 3\right)^{-1},
\end{aligned}
$$

(Eq. 6.6.24 of Ref. 11). These speeds are used to estimate the mode numbers needed to accurately compute the impulse response for any desired window of travel time about a selected peak. The $l$ th vertical mode, which is the Airy function, $\operatorname{Ai}\left(z / H-y_{l}\right)$, changes from oscillating to exponentially decaying when its argument is zero, i.e., $\check{z}=y_{l} H$. Since the zeros of $\mathrm{Ai}$ are negative real numbers, ${ }^{12}$ with $y_{l+1}>y_{l}$, the turning depths are positive and the turning depth of mode $l+1$ is greater than mode $l$.

\section{B. Approximate solution from parabolic approximation}

The sound-speed insensitive parabolic approximation ${ }^{9}$ yields accurate travel times, is efficient due to its split-step algorithm, and obeys reciprocity. It is important that reciprocity is obeyed because a proof ${ }^{1}$ for reciprocity is provided by the integral theorem of Helmholtz and Kirchhoff. In this paper the computational grids in depth and range are made sufficiently small to achieve convergence of the solution. The parabolic approximation yields estimates of $W_{i}\left(x_{s c}, z, \omega\right), i$ $=0,1$.

This algorithm for the parabolic approximation requires the depth of the bottom and the geoacoustic properties of the subbottom. The subbottom, starting at 5500-m depth, is constructed to absorb incident energy in the following way. The sediment thickness is $300 \mathrm{~m}$. The speed of sound at the top of the sediment divided by that at the bottom of the water column is 0.5 . This causes incident energy to refract downwards into the sediment. The density of the sediment and water are taken to be equal to minimize reflections at the interface. The attenuation in the sediment is $\alpha(f)=0.2 f(\mathrm{~dB} /$ $\mathrm{m}$ ), where $f$ is acoustic frequency in $\mathrm{kHz}$. The derivative of speed with depth is $0.001 \mathrm{~s}^{-1}$ in the sediment. The density of the lower basement layer is two and one-half times that of the water. The speed of sound in the basement is twice that at the bottom of the sediment layer. The attenuation in the basement is $\alpha(f)=0.5 f^{0.1}(\mathrm{~dB} / \mathrm{m})$. Diffracted regions will only be considered that do not interact with the bottom because the bottom is absent in the solution based on normal modes.

\section{Solution from ray approximation}

Regions of influence are compared with rays. The ray program, zray, and its eigenray finder have been described and successfully used to study acoustic propagation for many experiments. ${ }^{13,14}$ Its results agree with analytical solutions. Sound speeds used by the ray program are the same as those used by the parabolic approximation on its computational grid. Between grid points, the speed is obtained using a quadratic spline. The spline goes through each grid point without gradient discontinuities. ${ }^{13}$

\section{Results}

In Eq. (10), we set $c_{0}=1500 \mathrm{~m} \mathrm{~s}^{-1}$ and $a=1.2$ $\times 10^{-5} \mathrm{~m}^{-1}$. The speed increases by about $18 \mathrm{~m} \mathrm{~s}^{-1}$ per $1000 \mathrm{~m}$ of depth. Sidelobes are minimized in the time domain by applying a Hann taper in the frequency domain between $f_{c} \pm 20 \mathrm{~Hz}$ where the center frequency is $f_{c}$. The taper is zero at $f_{c} \pm 20 \mathrm{~Hz}$, yielding an effective bandwidth of $20 \mathrm{~Hz}$ and a time resolution of $\frac{1}{20}=0.05 \mathrm{~s}$.

Regions of influence are computed for the pulse having a travel time near $33.2 \mathrm{~s}$. For ray theory, the travel time of this pulse is $33.205 \mathrm{~s}$. The lower turning depth of the ray is about $1000 \mathrm{~m}$. It reflects once from the surface [Figs. 1(a) and $1(\mathrm{f})$ ]. At a pulse resolution of $0.05 \mathrm{~s}$, it is almost temporally resolved from its nearest ray arrival at $33.277 \mathrm{~s}$ that reflects twice from the surface [Figs. 1(a) and 1(f)]. These 

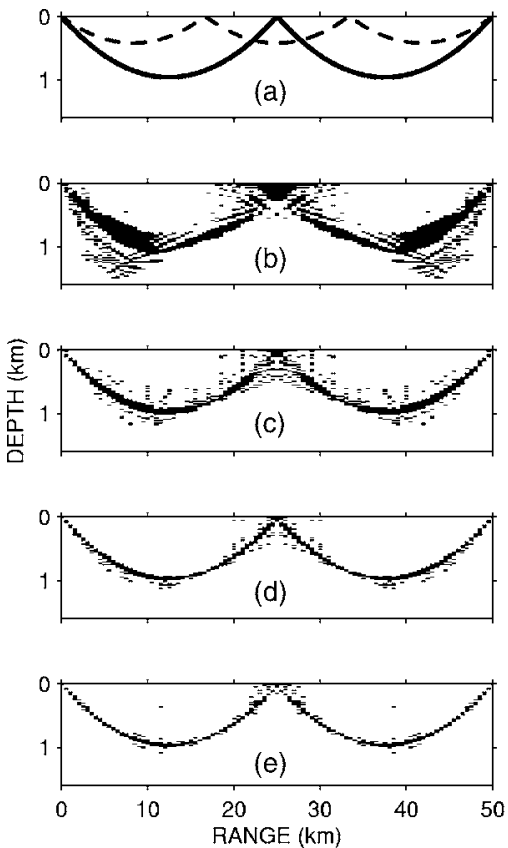
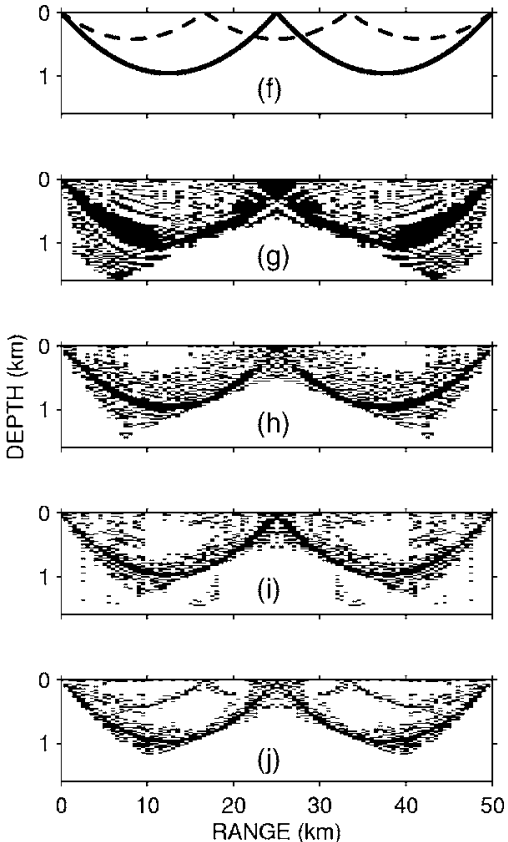

FIG. 1. (a, b) Two rays between source and receiver at $5-\mathrm{m}$ depth and 50-km separation compared with the constructive region of influence as a function of center frequency. The simulated signal has a bandwidth of $20 \mathrm{~Hz}$, and a center frequency of $100,500,1250$, and $2500 \mathrm{~Hz}$ in panels (b)-(e) and (g)-(j), respectively. Results are for the differential measure of influence of the first type (highest peak within the window of travel time) and the Huygens-Fresnel principle with inclination factor equal to unity [Eq. (8) for $k=1$ using Eq. (4)]. The left (right) column shows regions that contribute $0.9(0.99)$ of the amplitude of the peak within the window of travel time. The speed of sound varies with depth according to Eq. (10) with $c_{0}=1500 \mathrm{~m} \mathrm{~s}^{-1}$ and $a=1.2 \times 10^{-5} \mathrm{~m}^{-1}$. arrivals appear to be temporally resolved in the impulse responses of all solutions (not shown). As will be seen, however, a little energy from the later arrival leaks into the time window surrounding the earlier arrival.

The region of influence is estimated using the HuygensFresnel principle [Eq. (4)] at center frequencies of 100, 500, 1250 , and $2500 \mathrm{~Hz}$. We consider the energy arriving within $\pm 0.025 \mathrm{~s}$ of the peak corresponding to the arrival at $33.2 \mathrm{~s}$. Solutions for $W_{0}\left(x_{s c}, z, \omega\right)$ and $W_{1}\left(x_{s c}, z, \omega\right)$ are provided from normal modes. The fractional amplitude method is used to reconstruct the region of influence for fidelities of $f=0.9$ and $f=0.99$. Both ray paths are visible at $2500 \mathrm{~Hz}$ with a fidelity of 0.99 [Figs. $1(\mathrm{j})$ and $2(\mathrm{j})$ ]. At a fidelity of 0.9 , the ray that bounces twice from the surface is barely visible at this frequency [Figs. 1(e) and 2(e)]. At a fidelity of 0.99 and a center frequency of $1250 \mathrm{~Hz}$ (panel i), the region of influence has features that look unlike a ray. For example, sound hugs the surface over a distance of about $5 \mathrm{~km}$ where it reflects from the surface near $25 \mathrm{~km}$ of range. Other regions of space do not correspond to a ray path. At $2500 \mathrm{~Hz}$, departures from ray paths are more evident for regions constructed with a fidelity of 0.99 rather than 0.9 [Figs. 1(e), 1(j), 2(e), and $2(\mathrm{j})$ ]. Regions of influence look more raylike as the center frequency increases.

Constructive and destructive regions of influence are shaded as black and gray respectively in Figs. 1 and 2. They are most evident at $100 \mathrm{~Hz}$ where they occur as interleaving filaments. It is important to note that Figs. 1 and 2 do not show paths of sound between the source and receiver. Rather they shows constructive and destructive regions of influence.
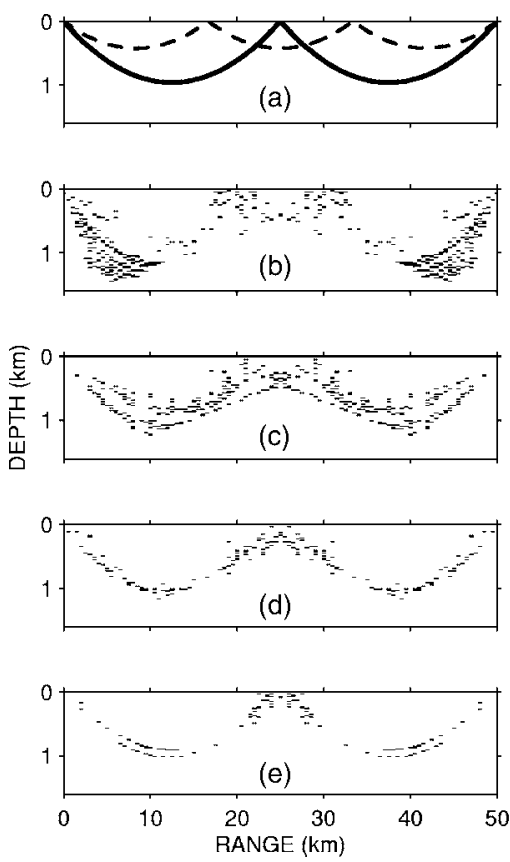
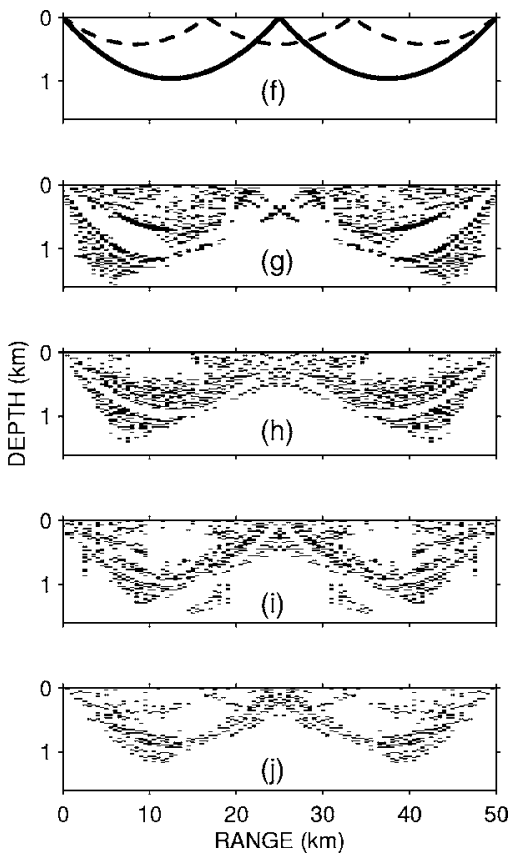

FIG. 2. Same as Fig. 1 except only destructive regions of influence are shown (gray) in (b)-(e) and (g) and (h). 


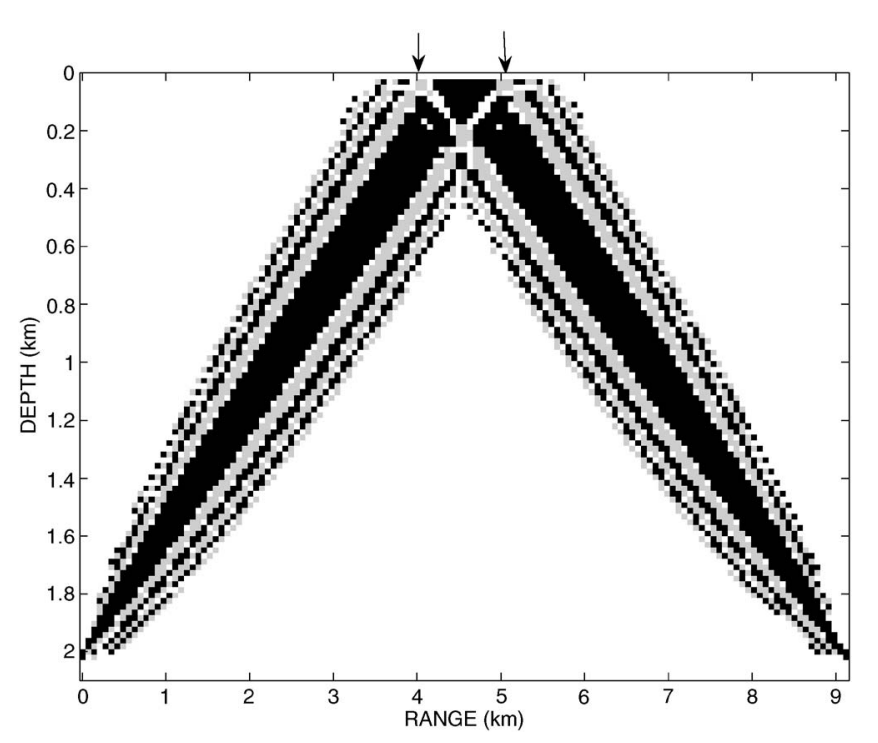

FIG. 3. Region of influence for propagation of path with one surface reflection in an otherwise homogeneous medium with wave speed $1.5 \mathrm{~km} / \mathrm{s}$. The source and receiver are at depths of $2 \mathrm{~km}$. To minimize sidelobes in the time domain, a Hann taper is applied in the frequency domain to the emitted signal between $100 \pm 40 \mathrm{~Hz}$. The taper is zero at 60 and $140 \mathrm{~Hz}$, yielding an effective bandwidth of $40 \mathrm{~Hz}$ and a time resolution of about $\frac{1}{40}=0.025 \mathrm{~s}$. The arrows indicate where two destructive paths of influence approach the surface. Adapted from Fig. 6 of Ref. 8.

For example, a region of influence shaded black at some range means that the signal from the source passing through an aperture on a screen corresponding to the black region has a significant influence on the measure of influence at the receiver. Where the signal goes before or after the aperture is a different question. ${ }^{7}$

The region of influence near the surface reflection shows a black triangular region [Figs. 1(b) and 1(g)]. It looks like that seen for a single reflection from a flat interface in homogeneous media (Fig. 3). For this later case, this triangle is carved out of the main region that corresponds to a specularly reflecting ray. There are two intense destructive regions of influence whose corresponding paths of influence arrive with phases one-half cycle greater than the signal from the main region. ${ }^{8}$ The coherent addition of waves from the main region and those corresponding to the destructive region of influence destructively interfere across the main region. This causes the triangular region at the surface that is carved out of the main region.

The intersection of the destructive region of influence with the surface corresponds to strong destructive paths of influence that seem to reflect from the surface at locations indicated by arrows in Fig. 3. However, they are caused by edge-diffracted rays described by the geometrical theory of diffraction. $^{7,8}$ In Fig. 3, we see other constructive and destructive regions of influence. Proceeding to the left from the leftmost arrow, we see alternating destructive and constructive regions corresponding to paths reflecting from the surface indicated in gray and black, respectively. The phases of the waves corresponding to these paths are $0.5,1,1.5,2$, and 2.5 cycles greater than the phase along the main region. ${ }^{8}$ Their received amplitudes decrease as the cycle difference increases with respect to the main region because they arrive later than the waves corresponding to the main region. Eventually, they arrive so late that their influence diminishes to zero within the selected window of travel time. A similar interpretation applies to the pattern for the region of influence for waves seen in the inhomogeneous waveguide [Figs. 1(b), 1(g), 2(b), and 2(g)]. These constructive and destructive regions of influence are entirely due to diffraction. Their influence diminishes as the center frequency increases (Figs. 1 and 2).

The differential measure of influence given by Eq. (8) has all the information needed to quantify significant contributions to the measure of influence. But we may not be interested in cases where adjoining constructive and destructive regions of influence lead to little net effect on the measure of influence. An interference filter can be applied to the differential measure of influence to guide intuition for
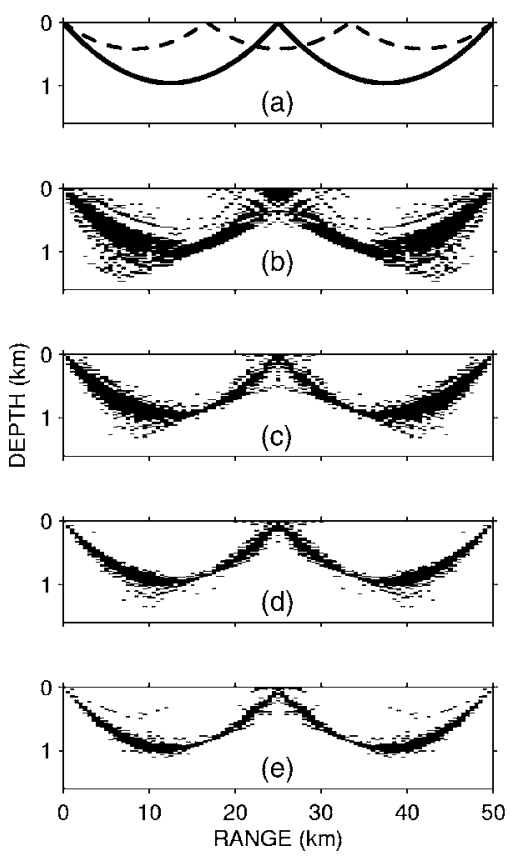
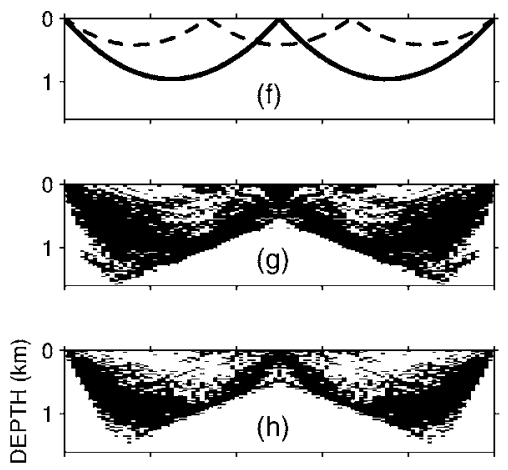

FIG. 4. Same as Figs. 1 and 2 except the interference filter is used. 

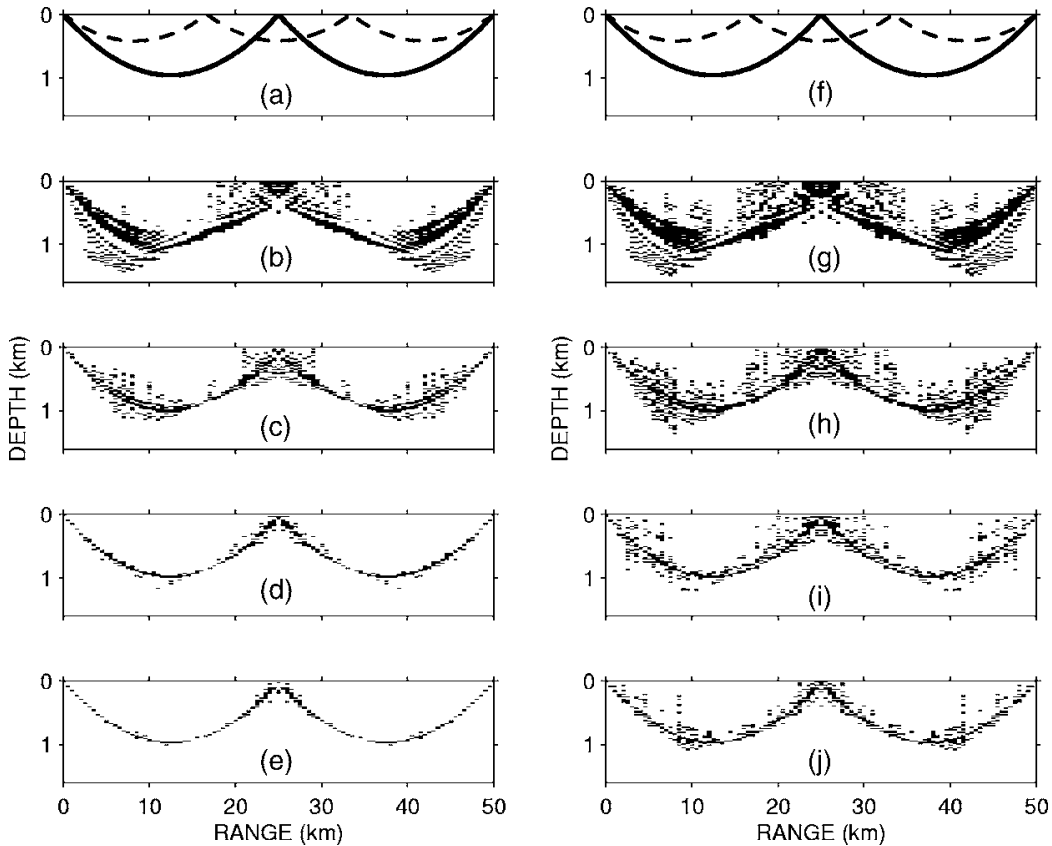

FIG. 5. Same as Fig. 1 except the measure of influence of the second type is used (energy of time series within window of travel time). regions of influence that are significant ${ }^{8}$ (Fig. 4), We see that the filtered region of influence becomes more raylike at higher frequency. The constructive paths of influence have more influence than the destructive paths of influence. This fact leads to the resemblance of the filtered and unfiltered regions of influence. The constructive paths of influence are partially canceled out by the destructive paths of influence, but not totally so. Waves corresponding to the main region of influence (specular reflection) have the largest amplitudes at the receiver. The strongest destructive path of influence is only one-half wavelength longer than the path for the main region. Thus, waves corresponding to the first destructive path of influence undergo about the same geometrical spreading loss as waves corresponding to the main region. However, the first destructive path of influence arrives later than waves from the main region, leading to a significant amount of its energy arriving later than the selected window of travel time. Thus, the net contribution from the main region and first destructive path of influence is won by waves corresponding to the main region. The same explanation applies to subsequent pairs of constructive and destructive paths of influence. The next pair of constructive and destructive paths of influence is dominated by the constructive path because it arrives less late than its destructive partner. The later the arrival, the less the influence in the selected window of travel time. If the constructive and destructive paths of influence arrived almost entirely within the selected window of travel time, their effects on the measure of influence would be insignificant because they would cancel each other. The interference filter is telling us that the constructive paths dominate the destructive paths of influence.

The region of influence looks about the same when we use the measure of influence of the second type (energy of time series within window of travel time) (Figs. 5 and 6). It is interesting that the weak ray path with two surface reflections does not appear with stronger contribution for the chosen values of fidelity. Its effect is still there [e.g., Figs. 5(i) and 6(i)] but is weak. Application of the interference filter yields a region of influence much like that seen for the first measure of influence (Fig. 7). As before, a center frequency of $2500 \mathrm{~Hz}$ looks much more raylike than at $100 \mathrm{~Hz}$.

We compare the region of influence at $100 \mathrm{~Hz}$ using the exact solution of the wave equation (normal modes) with the solution using the sound-speed insensitive parabolic approximation ${ }^{9}$ [Figs. 8(a) and 8(b)]. Panel (a) is the same as Fig. 4(b). Results are similar, which means that this parabolic approximation is accurate in this application. When we use the more accurate theory of diffraction based on the integral theorem of Helmholtz and Kirchhoff, ${ }^{1}$ results are very similar [Fig. 8(c)]. The reason for the similarity is that acoustic waves are propagating in a nearly horizontal direction. Thus the effect of the inclination factor ${ }^{1}$ in the Huygens-Fresnel theory is small. We have set the inclination factor to unity, which is evidently accurate for this case.

\section{LONGER DISTANCE PROPAGATION}

The region of influence at $2500 \mathrm{~Hz}$ is computed for a case that is identical to Sec. III D except the range between the source and receiver is $500 \mathrm{~km}$ instead of $50 \mathrm{~km}$. A temporally resolved peak is chosen from the normal mode solution having a travel time of $331.7625 \mathrm{~s}$. The neighboring peaks at 331.5625 and $331.9250 \mathrm{~s}$ are much further away than the pulse resolution of $0.05 \mathrm{~s}$ centered on the peak at $331.7625 \mathrm{~s}$. A raytrace shows the peak to correspond to a single ray leaving the source downward at an angle of $9.7166 \mathrm{deg}$ with a travel time of $331.755 \mathrm{~s}$ [Fig. 9(a)]. This differs slightly from the travel time of 331.7625 s computed via normal modes because of diffraction. The net region of influence is estimated using the Huygens-Fresnel principle and the fractional amplitude method as before. The fidelity of the fractional amplitude method is $f=0.9$. We use the measure of influence of the first type (highest peak in selected window of travel time). It looks more like a ray near the 

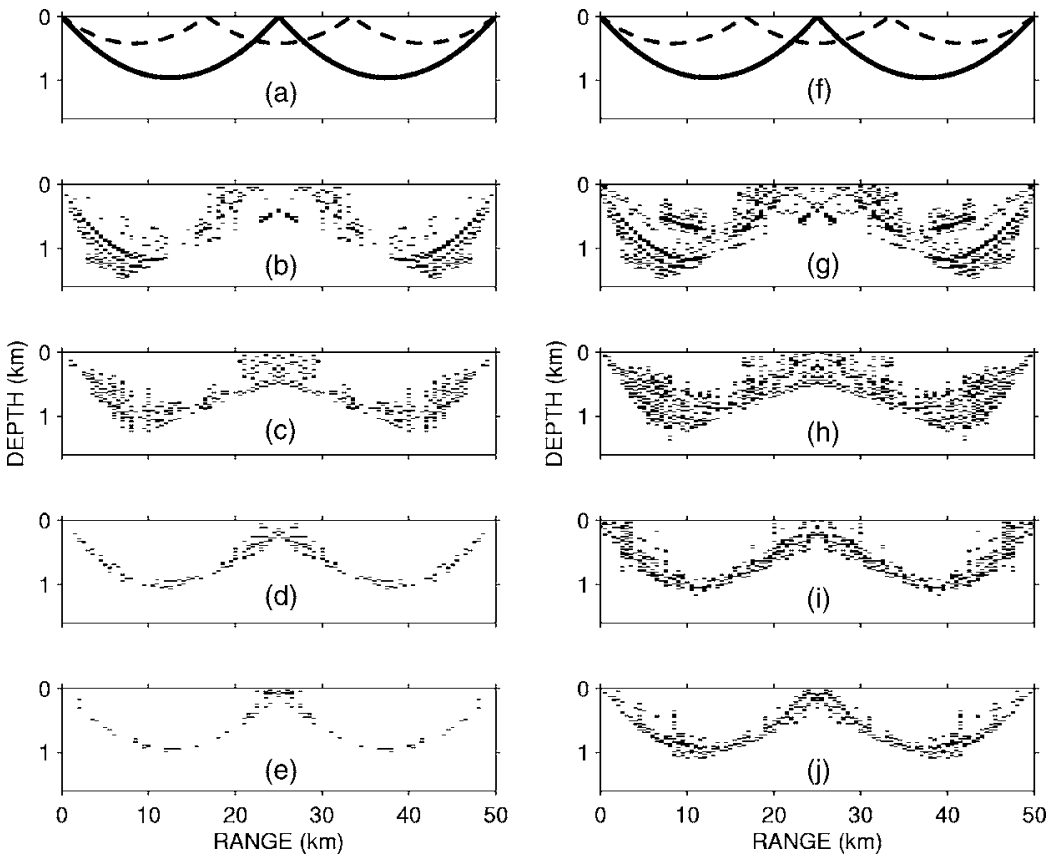

FIG. 6. Same as Fig. 2 except the measure of influence of the second type is used (energy of time series within window of travel time). source and receiver than in the central regions, where it exhibits many unraylike features such as diffuse regions near the surface [Fig. 9(b)]. Evidently, a center frequency of $2500 \mathrm{~Hz}$ is too low for the region of influence to look like a ray. If the fidelity of the reconstruction was increased to $f$ $=0.99$, the region of influence would appear even more unlike a ray, just as seen for the cases at a distance of $50 \mathrm{~km}$. Because the computations are lengthy at $2500 \mathrm{~Hz}$, calculations are not attempted at higher frequency. This simulation uses the first 1000 vertical modes at each acoustic frequency so as to guarantee an accurate solution for Helmholtz's equation.

\section{DISCUSSION AND CONCLUSION}

We investigated the regions of space that influence the waveform of a transient signal traveling between a source and receiver at 50 and $500 \mathrm{~km}$ in an idealized waveguide. At infinite frequency, these regions of influence coincide with one or more ray paths. At finite frequency, the regions of influence depart from the ray picture because of diffraction. At a distance of $50 \mathrm{~km}$, we found it necessary to go to a center frequency near $2500 \mathrm{~Hz}$ to obtain a region of influence that resembles a ray. The region of influence could be accurately calculated for any mix of approximations that include the sound-speed insensitive parabolic approximation and the Huygens-Fresnel theory of diffraction using an inclination factor of unity. These approximate solutions are very similar to solutions based on an exact solution of the wave equation via normal modes and the integral theorem of Helmholtz and Kirchhoff. This theorem automatically computes the correct inclination factor on each part of a screen. ${ }^{1}$ At a center frequency of $2500 \mathrm{~Hz}$, the region of influence
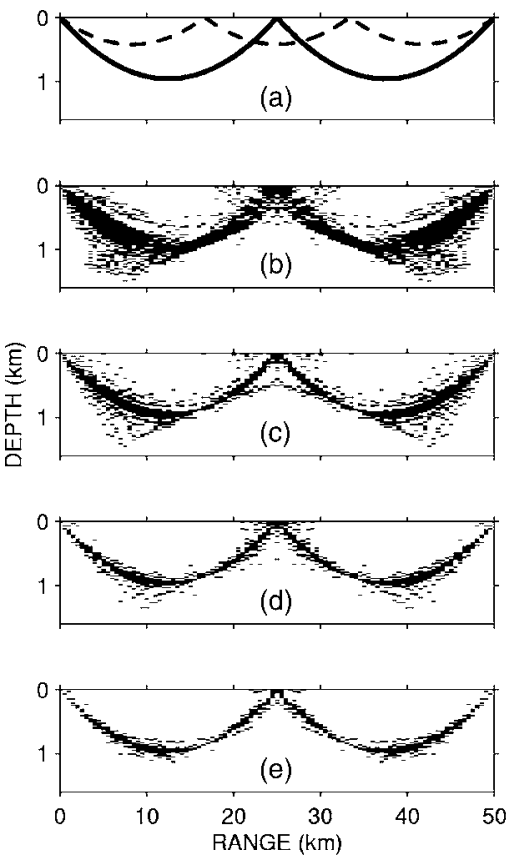
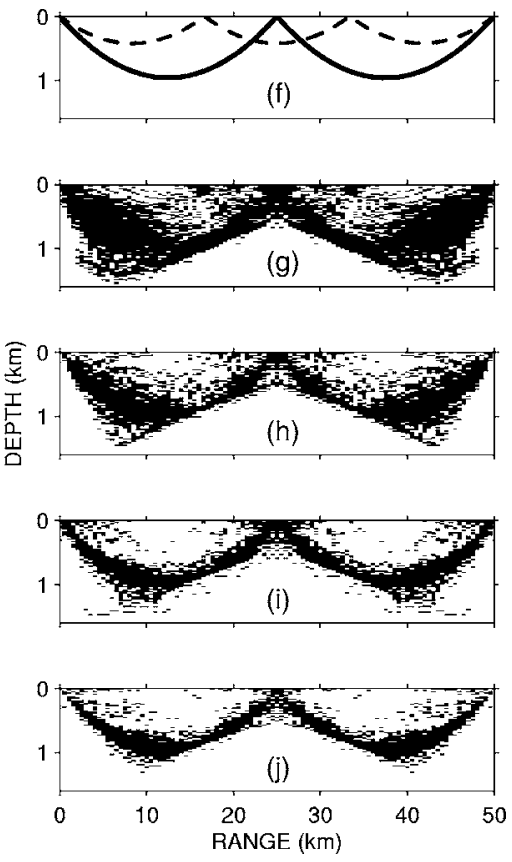

FIG. 7. Same as Figs. 5 and 6 except the interference filter is used. 

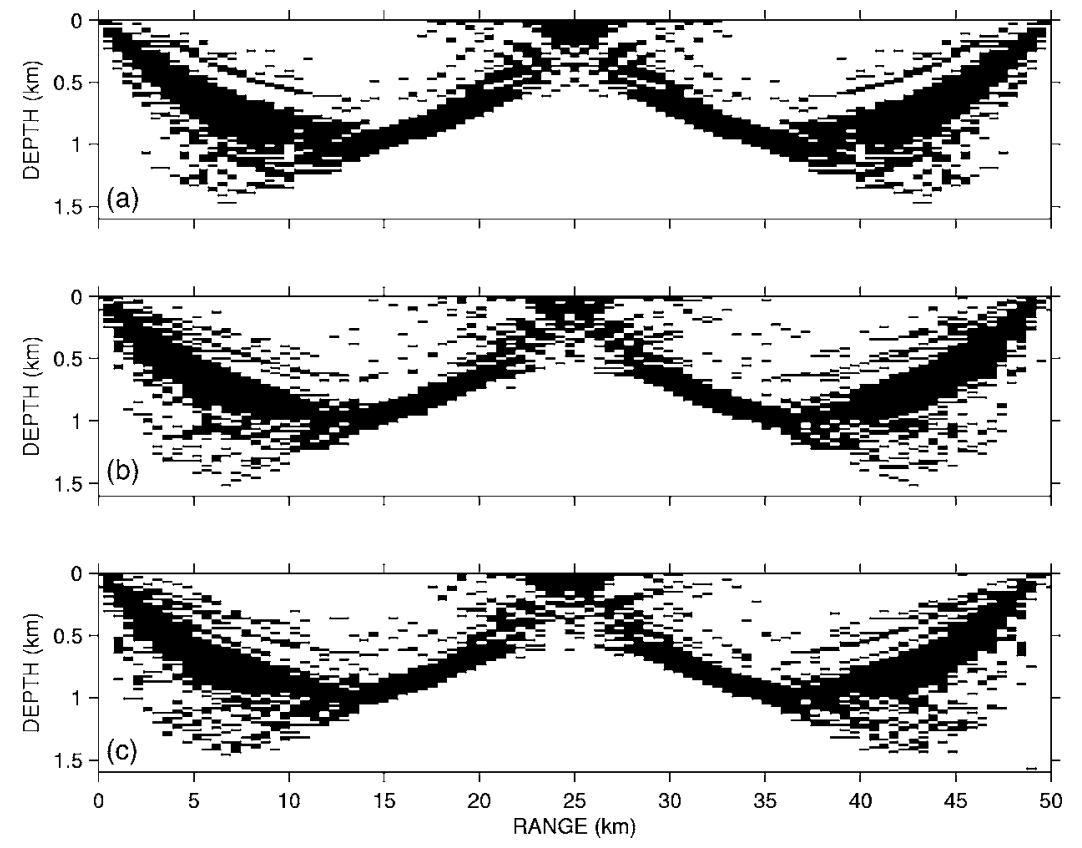

FIG. 8. (a) Region of influence computed with exact solution of wave equation (normal modes, $100 \mathrm{~Hz}$ center frequency, $20 \mathrm{~Hz}$ bandwidth) and Huygens-Fresnel principle for the measure of influence of the first type (highest peak in selected window of travel time). The interference filter has been applied so this is identical to Fig. 4(b). The region of influence is reconstructed with a fidelity of $f=0.9$. (b) Same except the wave equation is solved with the sound-speed insensitive parabolic approximation. ${ }^{9}$ (c) Same as (b) except effects of diffraction are computed from the integral theorem of Helmholtz and Kirchhoff. ${ }^{1}$ can look like a ray at a distance of $50 \mathrm{~km}$, but can exhibit nonraylike features at $500 \mathrm{~km}$. We conclude that both the center frequency and distance of wave propagation are factors contributing to departures from a ray path.

Regions of influence were previously computed using theories of diffraction based on the Huygens-Fresnel principle and the integral theorem of Helmholtz and Kirchhoff. ${ }^{8,15}$ These theories are implemented by solving the Green's function at a screen from both the source and receiver for many frequencies. This paper demonstrates that the sound-speed insensitive parabolic approximation ${ }^{9}$ yields Green's functions similar to those computed from exact solutions of the wave equation using normal modes. The previous computations for regions of influence ${ }^{8,15}$ are further validated here because the previous computations used the sound-speed insensitive parabolic approximation.
We found it possible to explain some features of the region of influence at low frequencies near $100 \mathrm{~Hz}$ with the idea of constructive and destructive paths of influence. It appears that some but not all paths of influence are caused by edge-diffracted rays. ${ }^{7,8}$ The constructive and destructive paths of influence significantly affect both measures of influence used in this study. Their effects grow smaller as the center frequency increases to $2500 \mathrm{~Hz}$. Since these paths are due to diffraction only, they must vanish at sufficiently high frequencies.

It appears that for some cases at 50-km range, the region of influence looks much like a ray at frequencies above $2500 \mathrm{~Hz}$, even when the fidelity of the measure of influence is reconstructed within $99 \%(f=0.99)$ of the complete mea-
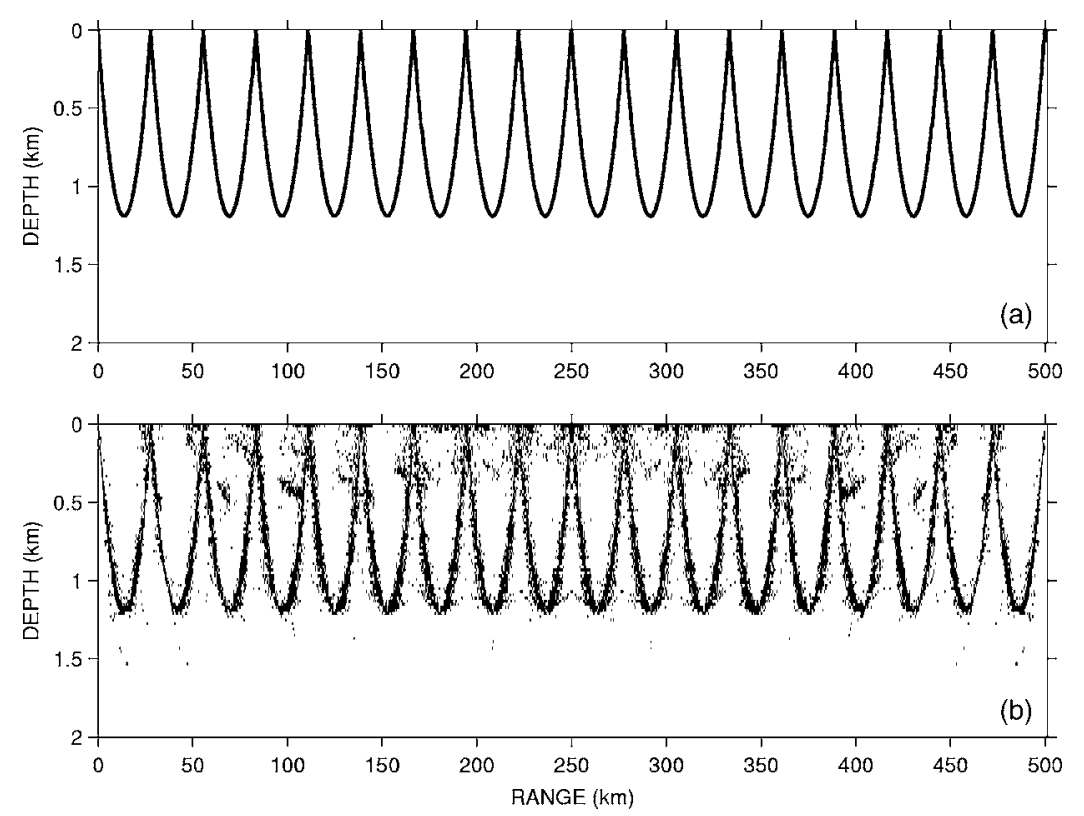

FIG. 9. (a) Ray between source and receiver at 5-m depths and 500-km distance. Sound travels this path in $331.755 \mathrm{~s}$. The sound speed field is the same as used for Fig. 1. (b) The corresponding region of influence for energy centered at $2500 \mathrm{~Hz}$ arriving within a pulse resolution of $0.05 \mathrm{~s}$ centered on the peak of the impulse response at $331.755 \mathrm{~s}$. The acoustic fields are computed exactly using normal modes. The region of influence is estimated using the interference filter, the HuygensFresnel principle, and the fractional amplitude method with a fidelity of $f=0.9$. The net region of influence has many un-ray-like features at this distance, despite the fact that the center frequency is large. 
sure of influence. At distances of $500 \mathrm{~km}$, it appears necessary that the center frequency be much larger to yield a region of influence that looks like a ray.

\section{ACKNOWLEDGMENTS}

This research was supported by the Office of Naval Research Contracts No. N00014-03-C-0155, and No. N0001406-C-0031, and by a grant of computer time from the DOD High Performance Computing Modernization Program at the Naval Oceanographic Office. I thank the reviewers and editor for their comments.

${ }^{1}$ Principles of Optics: Electromagnetic Theory of Propagation, Interference, and Diffraction, M. Born and E. Wolf, with contributions by A. Bhatia et al. (Cambridge U. P. Cambridge, 1999).

${ }^{2}$ A. W. Trorey, "A simple theory for seismic diffractions," Geophysics 35, 762-784 (1970).

${ }^{3}$ R. W. Knapp, "Fresnel zones in the light of broadband data," Geophysics 56, 354-359 (1991).

${ }^{4}$ M. Bruhl, G. J. O. Vermeer, and M. Kiehn, "Fresnel zones for broadband data," Geophysics 61, 600-604 (1996).
${ }^{5}$ B. R. Zavalishin, "Diffraction problems of 3D seismic imaging," Geophys. Prospect. 48, 631-645 (2000).

${ }^{6}$ J. Pearce and D. Mittleman, "Defining the Fresnel zone for broadband radiation," Phys. Rev. E 66, 056602 (2002).

${ }^{7}$ J. B. Keller, "Geometrical theory of diffraction," J. Opt. Soc. Am. 52, 116-130 (1962).

${ }^{8}$ J. L. Spiesberger, "Regions where transient signals are influenced between a source and receiver," Waves Random Media 16, 1-21 (2006).

${ }^{9}$ F. Tappert, J. L. Spiesberger, and L. Boden, "New full-wave approximation for ocean acoustic travel time predictions," J. Acoust. Soc. Am. 97, 2771-2782 (1995).

${ }^{10}$ J. Bowlin, "Generating eigenray tubes from two solutions of the wave equation," J. Acoust. Soc. Am. 89, 2663-2669 (1991).

${ }^{11}$ L. M. Brekhovskikh and Y. P. Lysanov, Fundamentals of Ocean Acoustics (Springer-Verlag, New York, 1991), p. 270.

${ }^{12}$ M. Abramowitz and I. A. Stegun, Handbook of Mathematical Functions (Dover, New York, 1972), p. 1046.

${ }^{13}$ J. B. Bowlin, J. L. Spiesberger, and L. F. Freitag, "Ocean acoustical raytracing software RAY," Woods Hole Oceanographic Technical Rept., WHOI-93-10, Woods Hole, MA. (1992).

${ }^{14}$ J. L. Spiesberger, "An updated perspective on basin-scale tomography," J. Acoust. Soc. Am. 109, 1740-1742 (2001).

${ }^{15}$ J. L. Spiesberger, "Locating where transient signals travel in inhomogeneous media," http://www.arxiv.org/abs/physics/0501162 (2005). 